\title{
Mobilité des impuretés ioniques dans le quartz
}

\author{
C. POIGNON, G. JEANDEL et G. MORLOT
}

Laboratoire Infrarouge Lointain, L.M.C.P.I. U.R.A. 809, Faculté des Sciences, BP. 239, 54506 Vandoeuvre-les-Nancy, France

\begin{abstract}
In order to produce very high quality oscillators of quartz, crystals which include a minimum of impurities are necessary. "Sweeping" is used in industry to purify synthetic crystals. An electric field is applied to a quartz sample at a given temperature to move ionic impurities nearer to the electrodes. After a lapse of time, the crystal is cooled and the field is removed and subsequently the extremities are either cut off or removed by polishing. The technique T.S.D. (Thermally Stimulated Depolarization) is used to better know the effects of "sweeping". Current densities are measured as a function of temperature. Two qualities of quartz, furnished by the S.I.C.N. ( Société Industrielle de Combustible Nucléaire à Annecy ) have been studied. Samples, purified or not, cut either perpendicular to the $\mathrm{Z}$ axis or to the $\mathrm{Y}$ axis, were extracted from various places in the crystal. The results obtained vary according to the origin of the studied samples and their treatment. In order to determine the influence of different parameters which occur at the time of "sweeping", we varied the intensity of the applied tension and the polarization time. Effects on the measurements of the thermoionic current were observed for a high quality sample.
\end{abstract}

\section{INTRODUCTION}

La présence de défauts et d'impuretés altère le comportement des résonateurs à quartz au cours du temps. Certaines impuretés et leurs implications jouent un rôle important dans cette dégradation. Lors de la croissance cristalline, des ions $\mathrm{Al}^{3+}$ se substituent à des ions $\mathrm{Si}^{4+}$. Pour conserver l'électroneutralité de l'édifice, des ions compensateurs ( alcalins, hydrogène) ou des trous sont présents près de chaque $\mathrm{Al}^{3+}$. L'alcalin ou l'hydrogène est en position interstitielle, le trou est localisé dans un oxygène adjacent à un ion 
substitutionnel. Diverses techniques sont employées pour observer ces défauts: par exemple, des mesures infrarouges [1,2], de relaxation diélectrique [3] et perte acoustique [4,5], de résonance paramagnétique électronique [6], de topographie $X$ [7].

Le quartz possède de larges canaux parallèlement à l'axe $Z$ ( c ) dans lesquels les impuretés interstitielles peuvent circuler. Le sweeping est utilisé industriellement comme méthode de purification. Un champ électrique est appliqué à l'échantillon à une température donnée selon la direction $Z$. Des ions vont se déplacer vers les électrodes sous l'effet de l'agitation thermique et du champ électrique: l'agitation thermique fournit l'énergie nécessaire à l'ion pour sortir du puits de potentiel que représente le "piège" $\mathrm{Al}^{3+}$ et le champ électrique dirige l'ion dans le canal vers l'électrode. L'échantillon est ensuite refroidi et le champ supprimé. Les extrémités polluées du cristal sont enlevées par une découpe ou un polissage.

Pour comprendre l'effet du sweeping, différentes méthodes électriques peuvent être utilisées, en particulier:

- T.S.P. ( Thermally Stimulated Polarization ) $[8,9]$.

- T.S.D. ( Thermally Stimulated Depolarization ) $[8,9]$.

Ces deux techniques ont déjà été employées. Le procédé T.S.D. nous semble le plus intéressant au niveau de l'exploitation. Des courants thermoioniques ont été mesurés et des pics de courant relatifs aux impuretés ont été observés.

\section{METHODE T.S.D.}

Le quartz est un matériau électriquement isolant, la conductivité est par conséquent ionique [10]. La méthode utilisée permet de mesurer des courants thermoioniques [11].

\subsection{Principe}

Un champ électrique appliqué à un échantillon crée soit un déplacement de charge d'une position d'équilibre à une autre, soit un déplacement de charge autour d'une position d'équilibre [12].

Un cristal est placé dans un cryostat à une température donnée $\mathrm{T}_{0}$. Il est soumis à une tension électrique $U_{0}$ pendant une durée $t_{0}$ ( voir schéma plus loin ). Les ions les moins liés au réseau vont se déplacer sous l'effet de l'agitation thermique et du champ électrique créé dans le matériau. Au bout de $t_{0}$, le cristal est refroidi jusqu'à la température de l'azote liquide. A $t_{1}$, la température est $77 \mathrm{~K}$, la tension $U_{0}$ est alors supprimée. La structure cristalline peut être considérée comme figée. Une remontée en température est effectuée ( d'une manière affine: $\mathrm{T}=\mathrm{bt}+\mathrm{c}$ ) au cours de laquelle un courant de dépolarisation est mesuré. 


\subsection{Théorie}

La relaxation par rotation de dipôles et le mouvement de charges d'espace sont étudiés car ce sont les phénomènes à l'origine du courant mesuré.

\subsubsection{Mouvement de charges d'espace}

En supposant que le champ dépolarisant est dû uniquement à des charges d'espace, la densité de courant s'écrit: [13,14]

$$
j(t)=-\frac{d P}{d t}
$$

et

$$
\mathbf{j}(t)=[\sigma] \mathbf{E}
$$

où $\quad[\sigma]$ est le tenseur de conductivité

E est le champ électrique dans le matériau

P est la polarisation.

$$
P_{i}=\varepsilon_{o} \chi_{i j} E_{j}
$$

où

$$
\begin{aligned}
& (\mathrm{i}, \mathrm{j}) \in\{\mathrm{x}, \mathrm{y}, \mathrm{z}\}^{2} \\
& {[\chi] \text { est le tenseur de susceptibilité. }}
\end{aligned}
$$

La conductivité suit la loi d'Arrhénius, donc elle est fonction de l'énergie d'activation. Dans notre cas, la mesure de courant est faite selon $Z$. Un pic de courant correspond à un maximum de $\mathrm{j}_{\mathrm{z}}$ à $\mathrm{T}_{\mathrm{m}}$ :

$$
\frac{\sigma_{z o} k T_{m}}{b \varepsilon_{0} \chi_{z z}\left(E_{z}-k T_{m}\right)} \exp \left[-\frac{E_{z}}{k T_{m}}\right]=1
$$

$E_{z}$ est l'énergie d'activation selon la direction $Z$ de la famille d'impuretés responsable de ce pic.

\subsubsection{Relaxation dipolaire}

Seule l'orientation des dipôles crée un champ dépolarisant. Les relaxations sont supposées non interactives. Pour un phénomène donné, on considère un temps de relaxation $\tau(T)[13,14]$. $\tau$ est fonction de $\tau_{0}$, une constante, et de $E$, une énergie d'activation. 
La densité de courant est définie par: $j(t)=-\frac{d P}{d t}$

Un pic de courant correspond à un maximum de $j(t)$ à une température $T_{m}$ :

$$
\frac{E b \tau_{o}}{k T_{m}^{2}} \exp \left(\frac{E}{k T_{m}}\right)=1
$$

\section{RESULTATS ET DISCUSSION}

\subsection{Caractérisation des échantillons et conditions expérimentales}

Des cristaux de quartz de haute qualité $\left(\mathrm{n}^{\circ} 272\right)$ et d'autres de qualité standard ( $\mathrm{n}^{\circ} 511$ ), fournis par la S.I.C.N. sont étudiés. Des mesures de courant thermoionique ont été réalisées sur des échantillons possédant des caractéristiques différentes. Dans de précédentes publications [13,14], plusieurs courbes ont été commentées. Dans ce papier, vont être présentées des courbes de densité de courant des échantillons, coupe Z, 511 et 272 non purifiés, de l'échantillon 272 swept sous vide. D'autre part, plusieurs courbes faites sur le 272 non swept dans des conditions expérimentales diverses seront aussi examinées.

La surface des échantillons étudiés diffère de l'un à l'autre, c'est pourquoi les mesures d'intensité de courant sont exprimées par unité de surface. Ainsi, les différentes courbes peuvent être comparées. L'épaisseur des cristaux testés (coupe Z) est $0,75 \mathrm{~mm}$.

Les électrodes choisies sont bloquantes. La remontée en température s'effectue à raison de $0,05 \mathrm{~K} / \mathrm{s}(=\mathrm{b})$ pour toutes les manipulations.

\subsection{Présentation et interprétation des courbes}

\subsubsection{Courbes des échantillons 272 et 511}

Les densités de courant des échantillons ( coupe Z ) non "swept" 272 et 511 sont présentées sur la figure 1 . Ils ont été tous deux extraits près du germe. La durée de polarisation à $T_{0}=350 \mathrm{~K}$ est de $t_{0}=14$ heures et l'intensité de la tension appliquée est $\mathrm{U}_{0}=1600 \mathrm{~V}$.

Les allures des deux courbes de la figure 1 sont différentes. La courbe de densité de courant de l'échantillon 511 présente un pic prédominant à $490 \mathrm{~K}$ et deux inflexions vers $400 \mathrm{~K}$ et $270 \mathrm{~K}$. Des pics de courant à $144 \mathrm{~K}, 211 \mathrm{~K}, 327 \mathrm{~K}$ et $425 \mathrm{~K}$ apparaissent sur la courbe du H.Q. 272; une inflexion vers $270 \mathrm{~K}$ est aussi observée. Les pics de basse température ne sont pas visibles pour l'échantillon 511. Les deux courbes sont tangentes en divers endroits. Vers $270 \mathrm{~K}$, une inflexion est observée pour les deux cristaux. La courbe de densité de courant de l'échantillon 272 présente un pic à $425 \mathrm{~K}$. A cet endroit, une inflexion, seulement est observée sur l'autre courbe; ceci est dû à la présence du pic intense à $490 \mathrm{~K}$. Globalement, l'aire soutendue à la courbe de densité de courant pour 
l'échantillon 511 est plus importante que celle pour le 272; ceci s'explique par le nombre d'impuretés plus élevé dans le 511 que dans le 272.

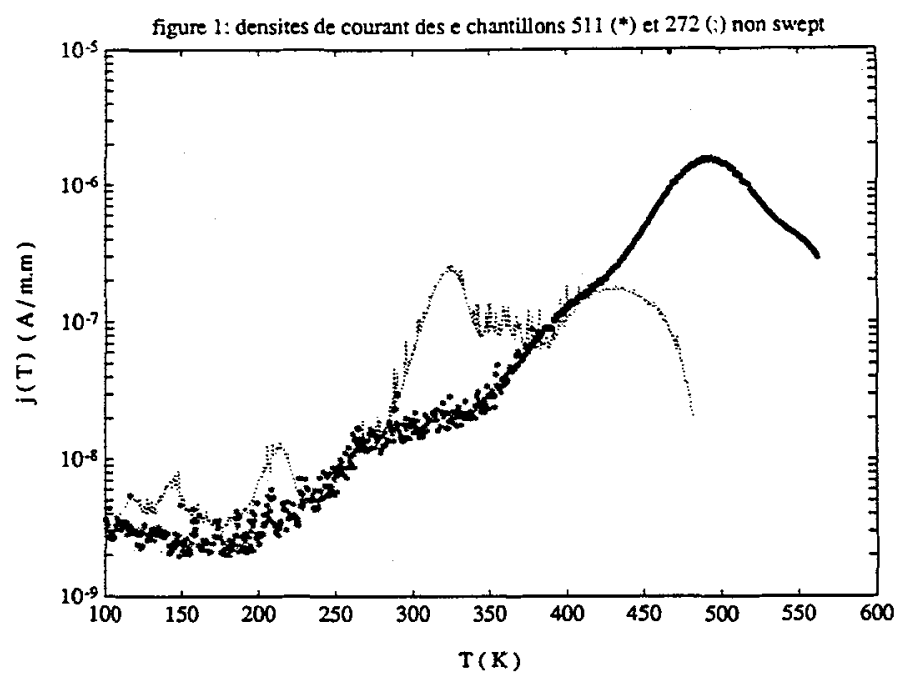

\subsubsection{Mesures successives sur l'échantillon 272 "swept"}

Les courbes $2 a, 2 b, 2 c$ et $2 d$ sur la figure 2 représentent des courbes de densités de courant d'un échantillon H.Q. 272 "swept" sous vide extrait près de l'anode. Ces mesures ont été faites dans les conditions indiquées ci-dessus, les unes après les autres. Il est à noter que, jusqu'à présent, seul cet échantillon a produit une variation du signal lors de mesures successives.

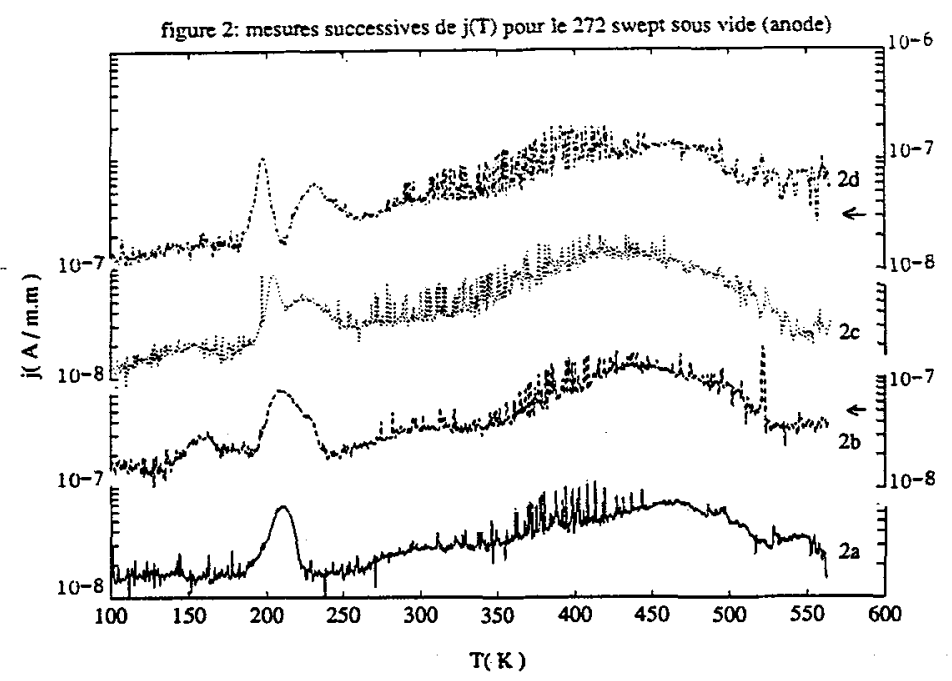

La courbe $2 \mathrm{a}$ possède deux maxima à $211 \mathrm{~K}$ et $466 \mathrm{~K}$. Une inflexion vers $300 \mathrm{~K}$ est visible. Cette figure représente la première mesure de courant 
thermoionique effectuée sur cet échantillon. Les trois autres courbes montrent une évolution au cours des différentes expériences. Le pic initialement centré à $211 \mathrm{~K}$ s'élargit sur la courbe $2 \mathrm{~b}$, puis se scinde en deux sur les courbes $2 \mathrm{c}$ et $2 \mathrm{~d}$. L'un des pics se décale progressivement vers des températures plus petites: $204 \mathrm{~K}$ pour $2 \mathrm{c}, 198 \mathrm{~K}$ pour $2 \mathrm{~d}$; l'autre vers des températures plus élevées: $225 \mathrm{~K}$ pour $2 \mathrm{c}$ et $233 \mathrm{~K}$ pour $2 \mathrm{~d}$. Le profil de la courbe évolue également pour les plus hautes températures. Parallèlement, la densité de courant augmente nettement entre la première mesure et les autres pour les températures élevées. L'inflexion vers $300 \mathrm{~K}$ est plus ou moins visible selon les cas.

\subsubsection{Variation de certains paramètres expérimentaux}

L'échantillon choisi est le H.Q. 272 non purifié, prélevé loin du germe. L'influence de la durée de polarisation $t_{0}$ à $350 \mathrm{~K}$ avec $\mathrm{U}_{0}=1600 \mathrm{~V}$ et l'influence de la tension de polarisation $U_{o}$ à $350 \mathrm{~K}$ avec $t_{o}=14 \mathrm{~h}$ sur les mesures de courant thermoionique ont été étudiées pour l'échantillon H.Q. nommé ci-dessus.

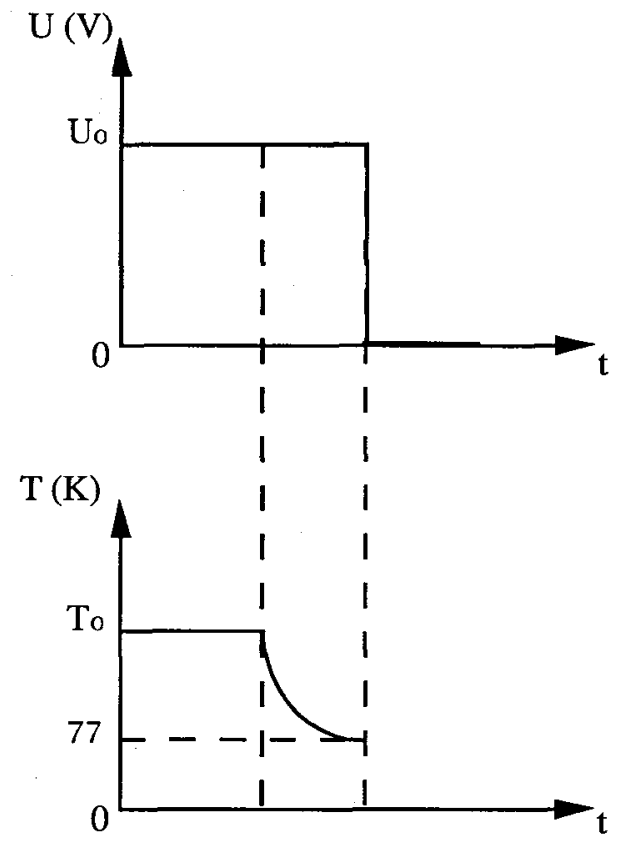

Schéma: représentation de l'évolution de la tension $U$ et de la température $T$ en fonction de $\mathrm{t}$.

3.2.3.1 Variation de la durée de polarisation $t_{0}$

L'intensité de la tension $U_{0}$. reste constante et égale à $1600 \mathrm{~V}$ et la température $T_{0}$ est $350 \mathrm{~K}$. 


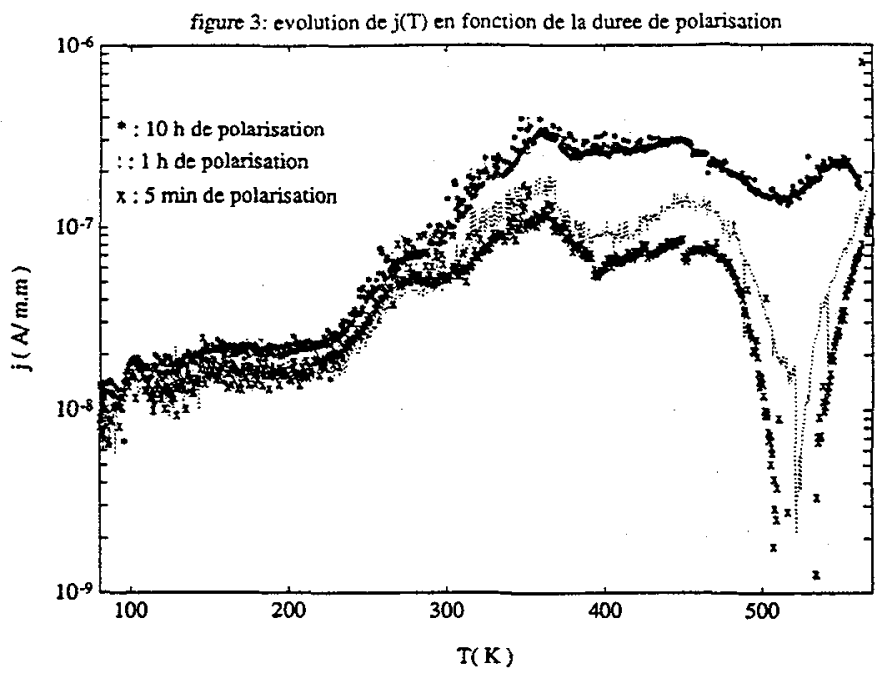

La densité de courant dépend de la durée de polarisation. Le nombre de charges déplacées augmente avec la durée de polarisation. Sur la figure 3 , trois courbes de densité de courant caractérisées par des $t_{0}$ différents $\left(t_{0}=5 \min , 1 \mathrm{~h}\right.$ et $10 \mathrm{~h}$ ) sont représentées. Les courbes correspondant à $t_{0}=5 \min$ et à $t_{0}=1 \mathrm{~h}$ sont très proches. Elles présentent, toutes deux, trois pics aux températures: $278 \mathrm{~K}, 363 \mathrm{~K}$ et $449 \mathrm{~K}$.. Globalement, quelle que soit $\mathrm{T}$, la densité de courant lorsque la polarisation dure $10 \mathrm{~h}$, est plus importante. Les trois pics observés précédemment figurent sur la courbe correspondant à $t_{0}=10 \mathrm{~h}$. De plus, un quatrième pic apparaît vers $550 \mathrm{~K}$ : des impuretés qui n'avaient pas eu le temps de bouger lorsque la polarisation durait 1 heure ou moins se sont déplacées. La différence d'amplitude est nettement visible pour les températures supérieures à $300 \mathrm{~K}$; l'amplitude du premier pic, elle, ne varie guère. Ce pic tend à être absorbé dans le suivant quand $t_{0}$ crôit. Les impuretés responsables de ce pic sont moins sensibles à la durée de polarisation que les impuretés responsables des autres pics. En effet, les charges se déplaçant à basse température sont très mobiles et sont peu influencées par les variations de $t_{0}$. Par contre, à haute température, les charges sont plus liées au réseau, il est plus difficile de les déplacer et elles sont davantage sensibles à la durée de polarisation.

\subsubsection{Variation de l'intensité de polarisation $\mathrm{U}_{\mathrm{o}}$}

La température $T_{0}$ est $350 \mathrm{~K}$ et la durée de polarisation $t_{0}$ égale à 14 heures.

La densité de courant dépend de l'intensité de polarisation. La figure 4 montre deux courbes de densité de courant: l'une réalisée avec $\mathrm{U}_{0}=200 \mathrm{~V}$, l'autre avec $\mathrm{U}_{0}=1600 \mathrm{~V}$. Quelle que soit la température, la densité de courant est plus faible après une polarisation de $200 \mathrm{~V}$ qu'après une de $1600 \mathrm{~V}$. L'inflexion à $278 \mathrm{~K}$ sur la courbe correspondant à $U_{0}=1600 \mathrm{~V}$ n'apparaît pas sur l'autre courbe. Peu 
d'impuretés ont bougé à $278 \mathrm{~K}$ avec $\mathrm{U}_{\mathrm{o}}=200 \mathrm{~V}$ et le pic résultant est totalement absorbé dans le pic suivant. Sur les deux courbes, trois maxima sont visibles à: $370 \mathrm{~K}, 450 \mathrm{~K}, 550 \mathrm{~K}$ pour l'une et $567 \mathrm{~K}$ pour l'autre. L'amplitude des pics centrés à $370 \mathrm{~K}$ et $450 \mathrm{~K}$ dépend fortement de la tension appliquée; par contre, celle du troisième pic paraît moins influencée par l'intensité de la tension. Un quatrième pic émerge à $615 \mathrm{~K}$ sur la courbe représentant la plus grande densité de courant, pas sur l'autre. La tension appliquée $U_{0}=200 \mathrm{~V}$ n'est sans doute pas suffisante pour déplacer la famille d'impuretés responsable de ce pic.

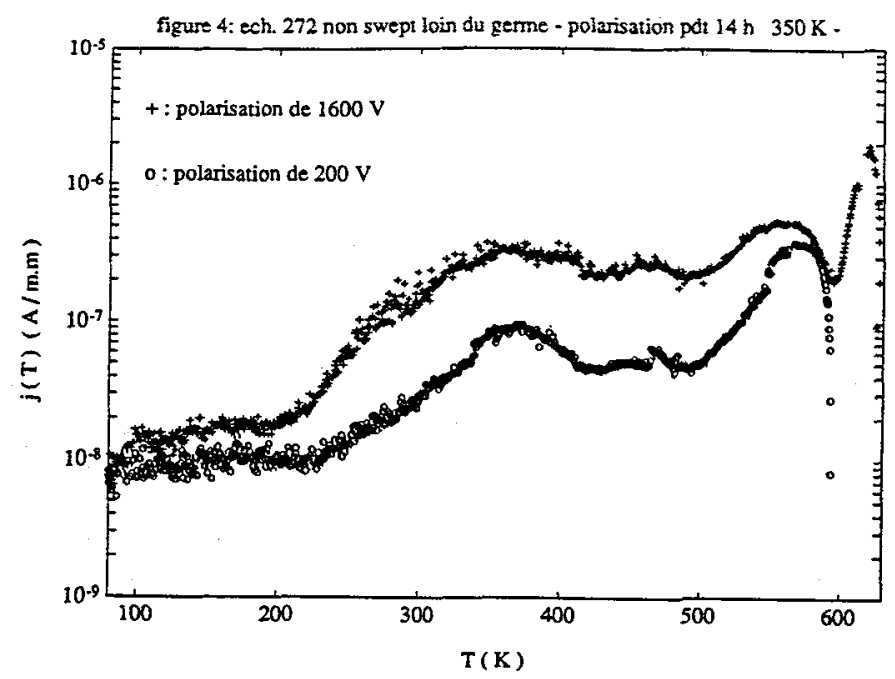

\section{CONCLUSION}

La méthode T.S.D. que nous avons utilisée permet de mesurer pour divers échantillons des courants thermoioniques dûs à des déplacements d'impuretés. Chaque pic de courant observé est caractéristique d'une famille d'impuretés ioniques. Les courbes de densité de courant de deux échantillons de qualité standard et de haute qualité ont des allures différentes: la densité de courant est fonction de la concentration en impuretés. Une évolution au cours de mesures successives faites sur l'échantillon "swept" sous vide a été observée. Par ailleurs, la densité de courant augmente avec la durée de polarisation d'une part et avec l'intensité de la tension appliquée d'autre part: ceci est d'autant plus sensible pour les pics obtenus à haute température qui correspondent à des déplacements d'impuretés très liées au réseau.

D'autres mesures sont en cours de réalisation pour compléter celles déjà faites. L'influence de la température de polarisation $T_{0}$ sera étudiée. Dans l'objectif de déterminer la nature des impuretés responsables des pics observés, des mesures de courant seront réalisées sur des échantillons contenant une impureté prédominante. 


\section{BIBLIOGRAPHIE}

[1] LIPSON H.G. \& KAHAN A., "Infrared characterization of aluminum and hydrogen defect centers in irradiated quartz", J. APPL. PHYS. 58 (2) (1985) 963-970.

[2] BACHHEIMER J.P. and DOLINO G., "Study of quartz sweeping in $\alpha$ and $\beta$ phases", PROC. 43th FREQ. CONT. SYMP.,(1989).

[3] PARK AND NOWICK, "Dielectric relaxation of point defects in $\alpha$-quartz" , PHYS. STAT. SOL. (a) 26, 617 (1974).

[4] FRASER D., PHYSICAL ACOUSTICS V ( ed. MASSON 1968), pp.59-108.

[5] MARTIN J.J., "Aluminum - related acoustic loss in AT-cut quartz crystals", J. APPL. PHYS. Vol. $56 \mathrm{n}^{\circ} 9$ (1984).

[6] CHATAGNON B., "La résonance paramagnétique électronique du centre $\mathrm{E}_{1}$ dans le quartz", Thèse I.N.P.L. Nancy (1986).

[7] DETAINT J., PHILIPPOT E., JUMAS J.C., SCHWARTZEL J., ZARKA A., CAPELLE B and DOUKHAN J.C, "Crystal growth, physical characterization and baw devices

applications of berlinite" , PROC. 39th FREQ. CONT. SYMP. (1975) pp. 234-246.

[8] MULLER P., "Relationship between thermally stimulated depolarization and conductivity", PHYS. STAT. SOL. (a) 23,165 (1974).

[9] MULLER P., "Electric relaxation currents in high-resistivity solids", PHYS. STAT. SOL. (a) 67,1,11-65 (1981).

[10] JAIN AND NOWICK, "Electrical conductivity of synthetic and natural quartz crystals", J. APPL. PHYS. 53(1), 477 (1982).

[11] BUCCI C., "Ionic thermocurrents in dielectrics", PHYSICAL REVIEW Vol.148 n² 816 (1966).

[12] JEANDEL G., WARIN A., MORLOT G., "Dielectric constant and I.T.C. measurements of $\mathrm{BaF}_{2}: \mathrm{Dy}^{3+} "$, PHYS. STAT. SOL. (a) 108, 185-187 (1988).

[13] JEANDEL G., WARIN A., POIGNON C., AUBRY J.P., MORLOT G.,"Mesure de courants thermoioniques dans le quartz", PROC. OF 6th EUROPEAN AND TIME FORUM, Noordwijk mars 1992, pp. 389-392.

[14] POIGNON C., JEANDEL G. \& MORLOT G., "Electric relaxation by movement of impurities in quartz" , PROC. OF 7th EUROPEAN AND TIME FORUM, Neuchâtel mars 1993, pp. 667-670. 